\title{
COLLECTIVE PROPERTIES OF ELECTRONS AND HOLES IN COUPLED QUANTUM DOTS
}

\author{
Guido Goldoni, Filippo Troiani, Massimo Rontani, Devis Bellucci, \\ and Elisa Molinari \\ INFM National Research Center on nanoStructures and bioSystems at Surfaces (S3) \\ Dipartimento di Fisica, Università degli Studi di Modena e Reggio Emilia, \\ Via Campi 213/A, 41100 Modena, Italy \\ Ulrich Hohenester \\ Institute für theoretische physik, Karl-Franzens-Universität Graz, \\ Universitäsplatz 5, 8010 Graz, Austria
}

\begin{abstract}
We discuss the properties of few electrons and electron-hole pairs confined in coupled semiconductor quantum dots, with emphasis on correlation effects and the role of tunneling. We shall discuss, in particular, exact diagonalization results for biexciton binding energy, electron-hole localization, magnetic-field induced Wigner molecules, and spin ordering.
\end{abstract}

\section{Introduction}

The similarity between quantum dots (QDs) and natural atoms, ensuing from the discrete density of states, is often pointed out (Maksym and Chakraborty, 1990; Kastner, 1993; Ashoori, 1996; Tarucha et al., 1996; Rontani et al., 2003). Shell structure (Ashoori, 1996; Tarucha et al., 1996), correlation effects (Bryant, 1987), and Kondo physics (Goldhaber-Gordon et al., 1998; Cronenwett et al., 1998) are among the most striking demonstrations. Aside from these similarities, and in addition to the huge technological interest in quantum systems which can be grown with very high control, there exist two main differences between natural and artificial atoms that make QDs particularly interesting also from a fundamental point of view. First, while in natural atoms Coulomb interactions are typically of the same order of the lowest energy single-particle gaps, in QDs these interactions can be made 
larger or much larger than the latter, due to the different scaling of kinetic and Coulomb energy contributions with respect to confinement length. Secondly, also magnetic field daily attainable in laboratories are associated to energy scales which are comparable with single-particle gaps; therefore, they can be used to manipulate the quantum states and, in particular, the ratio between kinetic and Coulomb contributions, so that in QDs one can reach regimes which are unattainable in natural atoms.

Coupled quantum dots, also called Artificial Molecules (AMs), extend to the molecular realm the similarity between quantum dots and artificial atoms (Kouwenhoven et al., 1990; Livermore et al., 1996; Blick et al., 1998; Brodsky et al., 2000; Rontani et al., 2001; Pi et al., 2001). Inter-dot tunneling introduces a kinetic contribution which has to be added to the QD confinement energy, and can be tuned with respect to it by structure engineering and by transverse magnetic fields. In addition, Coulomb interactions, both inter-dot and intra-dot, mix up in a very complicated way different electronic configurations (Slater determinants), the ratio between different contributions being a function, for example, of the external magnetic field strength and direction. Therefore, AMs constitute an interesting laboratory to study the physics of correlation. A specific aspect of AMs, with respect to single QDs, is that carrier-carrier interaction is tied with localization. Indeed, while kinetic energy favors delocalization over e.g. two QDs forming a diatomic AM, Coulomb interaction favors localization in opposite dots for charges of the same sign; the ratio between the two contributions and, therefore, the localized or delocalized character of the correlated state, is controlled by the tunneling energy.

From the theoretical point of view, highly correlated quantum systems are obviously a challenge. In many istances one is not allowed to use mean-field methods (Pfannkuche et al., 1993; Szafran et al., 2003). Reliable results can be obtained by a Configuration Interaction approach, where one uses the calculated single-particle states to form a large basis of Slater determinants to represent the Coulomb interaction. Obviously, this method has limitations in the number of free-carriers which can be treated and of single-particle levels included in the basis set. On the other hand, it provides accurate results which can be quantitatively compared with experiments and which represent a benchmark for more approximate methods.

In this work we review some theoretical results obtained for few electrons and electron-hole pairs in AMs. We specifically focus on the effects of carrier-carrier interactions, and the interplay of tunneling with other energy scales. All calculations are obtained by exact diagonalization 
methods of the Coulomb interaction. In a typical calculations we consider $N_{e}$ electrons and $N_{h}$ holes with the effective-mass Hamiltonian

$$
H=H_{e}+H_{h}+H_{e h},
$$

where

$$
\begin{aligned}
H_{\alpha} & =\sum_{i=1}^{N_{\alpha}}\left[-\frac{\hbar^{2}}{2 m_{\alpha}^{*}}\left(\nabla_{i}+\frac{e}{c} \mathbf{A}\left(\mathbf{r}_{i}^{\alpha}\right)\right)^{2}+V_{\alpha}\left(\mathbf{r}_{i}^{\alpha}\right)\right] \\
& +\frac{1}{2} \sum_{\substack{i, j=1 \\
i \neq j}}^{N_{\alpha}} \frac{e^{2}}{\epsilon^{*}\left|\mathbf{r}_{i}^{\alpha}-\mathbf{r}_{j}^{\alpha}\right|^{2}}+g^{*} \mu_{B} \vec{\sigma} \cdot \mathbf{B}, \\
H_{e h} & =-\sum_{i=1, j=1}^{N_{e}, N_{h}} \frac{e^{2}}{\epsilon^{*}\left|\mathbf{r}_{i}^{e}-\mathbf{r}_{j}^{h}\right|^{2}},
\end{aligned}
$$

with $\alpha=e, h$. Here $m_{\alpha}^{*}$ and $\epsilon^{*}$ are the effective mass and dielectric constant, respectively, $g^{*}$ is the effective giromagnetic factor, $\mu_{B}$ the Bohr magneton, $\mathbf{A}$ is the vector potential generating the magnetic field $\mathbf{B}$, and $e=|e|$; all parameters are taken for the AlGaAs class of materials. Equations (2) neglect non-parabolicity and spin-orbit effects, but otherwise describe samples with realistic properties, such as layer width and finite band offsets, by means of the effective potentials $V_{\alpha}(\mathbf{r})$. Typical samples that we shall consider consist of two identical QDs obtained from symmetric coupled quantum wells, grown, say, along the $z$ direction, of width $L_{W}$, separated by a barrier $d$, and with band-offset $V_{0}$ between wells and barriers. The lateral confinement can be often taken as a two-dimensional (2D) parabolic potential characterized by an energy $\hbar \omega_{0}$ which may assume rather different values, depending whether QDs are obtained by gating or by etching of a 2D electron/hole gas, by self-assembly, etc. The lateral confinement energy $\hbar \omega_{0}$ is typically of the order 1-10 meV and often much weaker than the confinement along the growth direction.

Our numerical approach consists in mapping the single-particle terms $H_{\alpha}$ in a real-space grid, leading to a large sparse matrix which is diagonalized by Lanczos methods. Single-particle spin-orbitals thus obtained are then used to build a basis of Slater determinants for the $N$-particle problem, which is then used to represent the two-body term, in the familiar Configuration Interaction approach. Coulomb matrix elements are calculated numerically. The ensuing matrix, which can be very large, is again sparse and can be diagonalized via the Lanczos method (Lehoucq et al., ). 
The paper is organized as follows. In Sec. 1.2 we discuss electronhole complexes, and particularly biexciton binding and localization. In Sec. 1.3 we discuss the phase diagram of few electrons in a magnetic field. In particular, in Sec. 1.3.1 we consider the effects of tunneling on few electrons in a large vertical field, where carriers are localized in the so-called Wigner molecule. Finally, in Sec. 1.3.2 we show how spin-ordering of two electrons can be manipulated by a magnetic field of arbitrary strength and direction.

\section{Electron-hole complexes in AM}

In this section we are concerned with the biexciton states in two identical vertically-coupled QDs: the main focus is on the effects of the Coulomb-induced interdot correlations, which are investigated as a function of the barrier width $d$ (Troiani et al., 2002). The features of the biexcitonic states are shown to critically depend on the detailed balance between three phenomena: $(i)$ the interdot tunneling, which is a singleparticle feature, tending to delocalize the carriers and to spread them over the artificial molecule; (ii) the homopolar Coulomb interactions, which is a few-particle effect, resulting in spatial correlations between the two electrons (holes) that tend to minimize the repulsion energy by localizing the identical carriers in opposite dots; ( $i i i)$ the heteropolar Coulomb interactions, that induce spatial correlations between electrons and holes in order to maximize their overlap. Interestingly enough, the interplay between these trends can be widely tuned, either by modifying the structural parameters of the dots or by applying external fields, in order to induce non-trivial behaviours already at the few-particle level.

In the following we fix all the physical parameters of the AM but the interdot distance $d$ in order to explore the different coupling (tunneling) regimes. At the smallest interdot distance, the symmetric-antisymmetric (S-AS) splitting $2 t^{(e, h)}=\epsilon_{A S}^{(e, h)}-\epsilon_{S}^{(e, h)}$ is maximized, where $\epsilon_{S}\left(\epsilon_{A S}\right)$ is the energy of the single-particle S (AS) level. For the sample of Fig. 1 , for example, where $d$ is varied in the range $1 \div 3 \mathrm{~nm}$, the tunneling splitting amounts to about 12 and $6 \mathrm{meV}$ for the electrons and the holes, respectively, at $d=1 \mathrm{~nm}$. As a result, both electrons are frozen in the $\mathrm{S}$ state and the spatial distribution of each electron is uncorrelated both with respect to the other electron position and to that of the two holes. Due to their smaller effective mass, the holes tunnel less efficiently than the electrons $\left(t^{(h)}<t^{(e)}\right)$ : therefore the interdot spatial correlation, which requires the occupation of the AS states, is favored and acts in such a way that the carriers are always localized in different dots. This is shown in the left-hand insets of Fig. 1(b-d), where we plot 

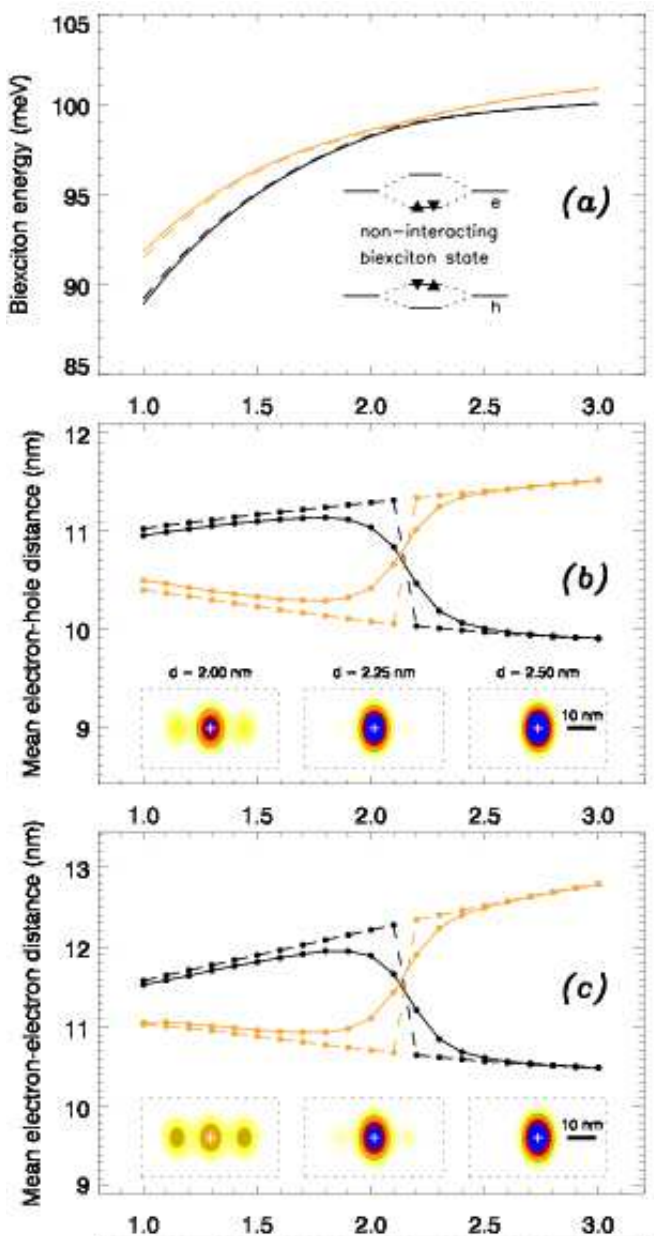

Figure 1. Biexciton energies (a) and mean distances between carriers (b-d) as a function of the interdot distance $d$. The plots refer to the four states lowest in energy, with even (continuous lines) or odd (dotted lines) parity. The insets (b-d) show the dependence of the pair-correlation functions on the relative position $\mathbf{r}=\mathbf{r}_{1}-\mathbf{r}_{2}$ of

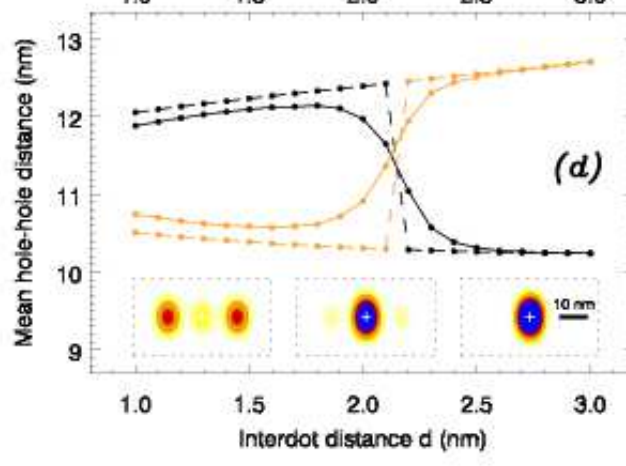

the two carriers: in particular, we take $y=0$ and show the dependence on the $z$ (horizontal axis) and $x$ (vertical axis) coordinates. The two verticallycoupled GaAs/AlGaAs QDs are modeled by means of a confinement potential which is double-well like in the growth direction (the well width and band offsets are $L_{W}=10 \mathrm{~nm}$ and $V_{0}^{(e, h)}=400,215 \mathrm{meV}$ respectively) and parabolic in the plane $\left(\hbar \omega_{0}^{(e, h)}=\right.$ 20,3.5 meV, $\left.m_{(e, h)}^{*}=0.067,0.38 m_{0}\right)$. 
the dependence of the pair-correlation on the relative position between the carriers. By progressively increasing the barrier width, the effects of the homopolar Coulomb interactions between the carriers continuously increase, whereas electrons and holes are not correlated with each other. In this kind of configuration, resulting in a factorized wavefunction $\psi\left(\mathbf{r}_{e 1}, \mathbf{r}_{e 2}, \mathbf{r}_{h 1}, \mathbf{r}_{h 2}\right) \simeq \phi^{(e)}\left(\mathbf{r}_{e 1}, \mathbf{r}_{e 2}\right) \phi^{(h)}\left(\mathbf{r}_{h 1}, \mathbf{r}_{h 2}\right)$, the probability of having double occupancies (two electrons or two holes) in each dot is strongly suppressed, i.e., the two excitons are localized in different dots. In approaching the weak-coupling regime, the biexciton groundstate undergoes a rapid transition towards a maximally correlated configuration, where all the carriers are localized in the same dot (see the right-hand insets of Fig. 1(b-d)). The reason why this arrangement is energetically favored as compared to the previously discussed one is entirely related to the in-plane correlations between the carriers. In fact, due to the substantial symmetry of the electron and hole wavefunctions, the Coulomb energy of the two configurations is the same for both types of particles in the mean-field limit: the increase in the Coulomb repulsion arising from the stronger degree of localization is cancelled by that of the Coulomb attraction. The occupation of the higher Fock-Darwin states, however, gives rise to additional (in-plane) spatial correlations, such as the ones that cause the biexciton binding energies in single QDs. This kind of interaction, whose closest classical analogue is an induced dipole-induced dipole force, is a short range one and is therefore uneffective as far as the two excitons are localized in different dots. In Fig. 1 we plot the energy levels [panel (a)] and the average distance between each pair of carriers [panels (b)-(d)] for the four lowest biexciton states: the continuous (dotted) lines correspond to the two states of even (odd) symmetry. Two features emerge from the plots: $(i)$ the transition between the weakly- and the highly-correlated configurations occurs quite abruptly for $d \simeq 2 \mathrm{~nm}$; $(i i)$ in a limited interval around this value each of the four states swaps its features with state of equal symmetry, and correspondingly an anticrossing occurs between their energy levels.

The interdot correlation is therefore seen to play a crucial role in determining the carrier localization in artificial molecules: these exhibit a fully three-dimensional nature, where novel behaviors occur as compared to the single dots. Indeed, such features have to be taken into account within the design of exciton-based quantum computation schemes and devices (Troiani et al., 2000), where the double occupancies are known to spoil the required (tensorial) Hilbert-space structure. 


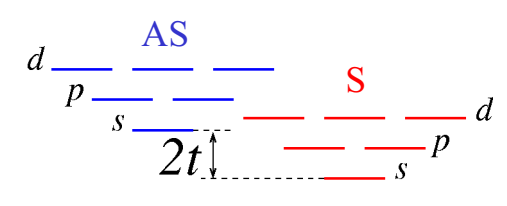

(a)

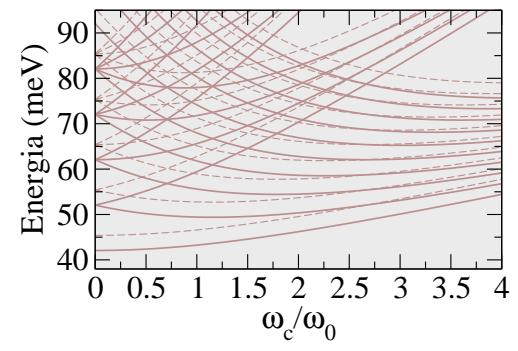

(b)

Figure 2. (a) Sketch of the energy levels in a 2D parabolic potential for an AM at zero magnetic field. $s, p, d$ label the value of the single-particle magnetic moment consistent with the corresponding atomic notation. S and AS stand for Symmetric and AntiSymmetric levels arising from two coupled QDs with tunneling energy $2 t$. (b) Fock-Darwin levels (Eq. 3) for an AM with $L_{w}=10 \mathrm{~nm}, d=3 \mathrm{~nm}, V_{0}=300$ $\mathrm{meV}$, and $\hbar \omega_{0}=10 \mathrm{meV}$.

\section{Interacting electrons in $\mathbf{A M}$ in a magnetic fields}

In this section we shall consider the effects of a magnetic field of arbitrary strength and direction on the few-electron states in AMs. Let us first summarize a few well-known effects of a field which is parallel to the axis of the AM.

Figure 2(a) shows the single-particle levels at zero field, separated by $\hbar \omega_{0}$, ensuing from the $2 \mathrm{D}$ parabolic potential of the in-plane confinement. The two sets of shells are separated by the tunneling energy. As a vertical field is switched on, the Hamiltonian can still be analytically solved (Jacak et al., 1998), and gives rise to the Fock-Darwin (FD) levels

$$
\varepsilon_{n m}^{i}=\varepsilon_{i}+\hbar \Omega(2 n+|m|+1)-\left(\hbar \omega_{c} / 2\right) m .
$$

Here $n$ is the principal quantum number describing the radial distribution of the wavefunction, and $m$ the azimuthal quantum number labelling the angular momentum which is conserved in this cylindrically symmetric configuration. The oscillator frequency $\Omega=\left(\omega_{0}^{2}+\omega_{c}^{2} / 4\right)^{1 / 2}$, with the cyclotron frequency $\omega_{c}=e B / m^{*} c$, shows the competition between the confinement energy gaps, $\hbar \omega_{0}$, and the field-induced gaps, $\hbar \omega_{c}$. The energy $\varepsilon_{i}$ is the confinement energy along the growth direction. For AMs with sufficiently narrow symmetric quantum well we can limit our considerations to two levels, which are the ground $\mathrm{S}$ and AS states $(i=\mathrm{S}$, 


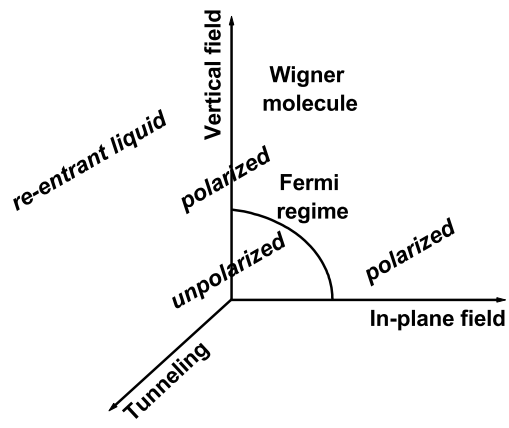

Figure 3. Sketch of the ground state phase diagram for an AM with respect to field strength and direction, and tunneling.

AS). The Fock-Darwin states for an AM are shown with lines in Fig. 2(b). At zero field one recovers the $s, p, \ldots$ shells of Fig. 2(a). At finite field, the $\pm m$ orbital degeneracy splits, and at large fields the levels with highest $m$ tend to form highly degenerate Landau levels.

Figure 3 shows a qualitative sketch of the ground state phase diagram of few-electrons in a AM which will be discussed in the next sections. Let us focus, for the time being, on the vertical field effects. When a field is applied parallel to the growth axis, the effect is to squeeze the states in the QD plane. By doing so, Coulomb energy increases and, at sufficiently large field, the system becomes spin-polarized in order to gain in exchange energy. At even larger fields, kinetic energy is quenched, since all single-particle levels tend to become degenerate [Fig. 2(b)], and correlation dominates the system. Accordingly, the electron system first becomes a spin-polarized confined Fermi sea, laterally delocalized over the QDs. Then, at very high fields, the systems transforms to a confined Wigner crystal, in which the carriers are localized in the plane of the QD; the latter regime is often called Wigner molecule (Maksym et al., 2000).

There are two obvious directions along which the phase diagram of a AM can be extended: the tunneling energy and an in-plane component of the magnetic field, as shown in Fig. 3. These two regions will be discussed next. 


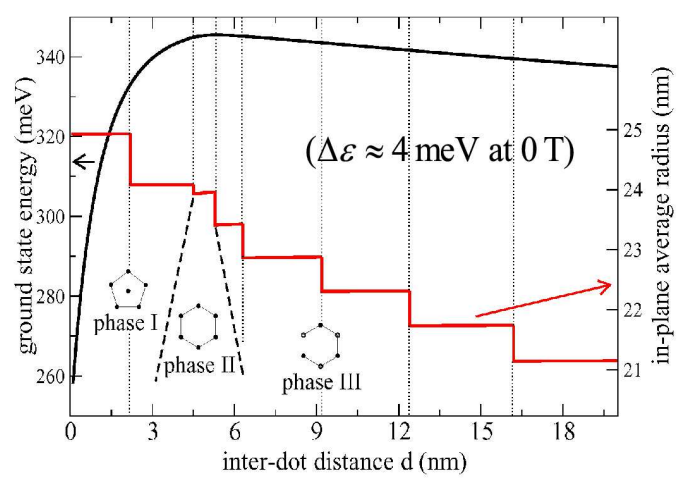

Figure 4. Ground-state energy (left axis) and in-plane average radius $\langle\varrho\rangle$ (right axis) vs inter-dot distance $d$ for six electrons at $B=25 \mathrm{~T}$. Sample parameters are: $\hbar \omega_{0}=$ $3.7 \mathrm{meV}, V_{0}=250 \mathrm{meV}$, and $L_{W}=12 \mathrm{~nm}$. Insets show the electron arrangements in the different phases.

\subsection{Wigner molecules and tunneling}

As an example of the effects of tunneling in the high field regime, we show in Fig. 4 the calculated ground-state energy vs the inter-dot distance $d$ for six electrons (Rontani et al., 2002). According to the previous discussion, we assume that at this field carriers are spin polarized; furthermore, at the high field considered here, electrons in a single QD are expected to be localized, and quantum fluctuation to play a minor effect. In the inset we also show the geometrical configuration of the localized carriers which will be discussed in more detail in Fig. 5. At small distances the energy increases with $d$ (phase I), because the kinetic energy exponentially grows due to the progressive localization of the wavefunction into the dots: electrons occupy only S orbitals, whose energies increase. At these small $d$ the AM behaves as a single $\mathrm{QD}$, and electrons sit at the vertices of a centered pentagon, which minimizes the Coulomb energy, as predicted by a classical static calculation (Partoens et al., 1997). There is another obvious regime: when the barrier is large, the six electrons sit, three per QD, at the vertices of two triangles staggered by $60^{\circ}$ (Phase III). Close to $d=5 \mathrm{~nm}$ inter-dot tunneling stabilizes Phase II, in which electrons sit at the vertices of an hexagon. The figure also shows the calculated average radius $\langle\varrho\rangle[\varrho \equiv(x, y)]$; Fig. 4 shows that these states are incompressible, in the same sense as Laughlin's states of the FQHE (Laughlin, 1983). Indeed, varying $d$ acts like an external pressure applied in the $z$ direction, forcing the wavefunction to 
change: however, due to a cusp-like structure of the energy spectrum (Maksym and Chakraborty, 1990), this happens only in a discontinuous way, except for Phase II. We show below that Phase II has very special properties and is stabilized by tunneling fluctuations.

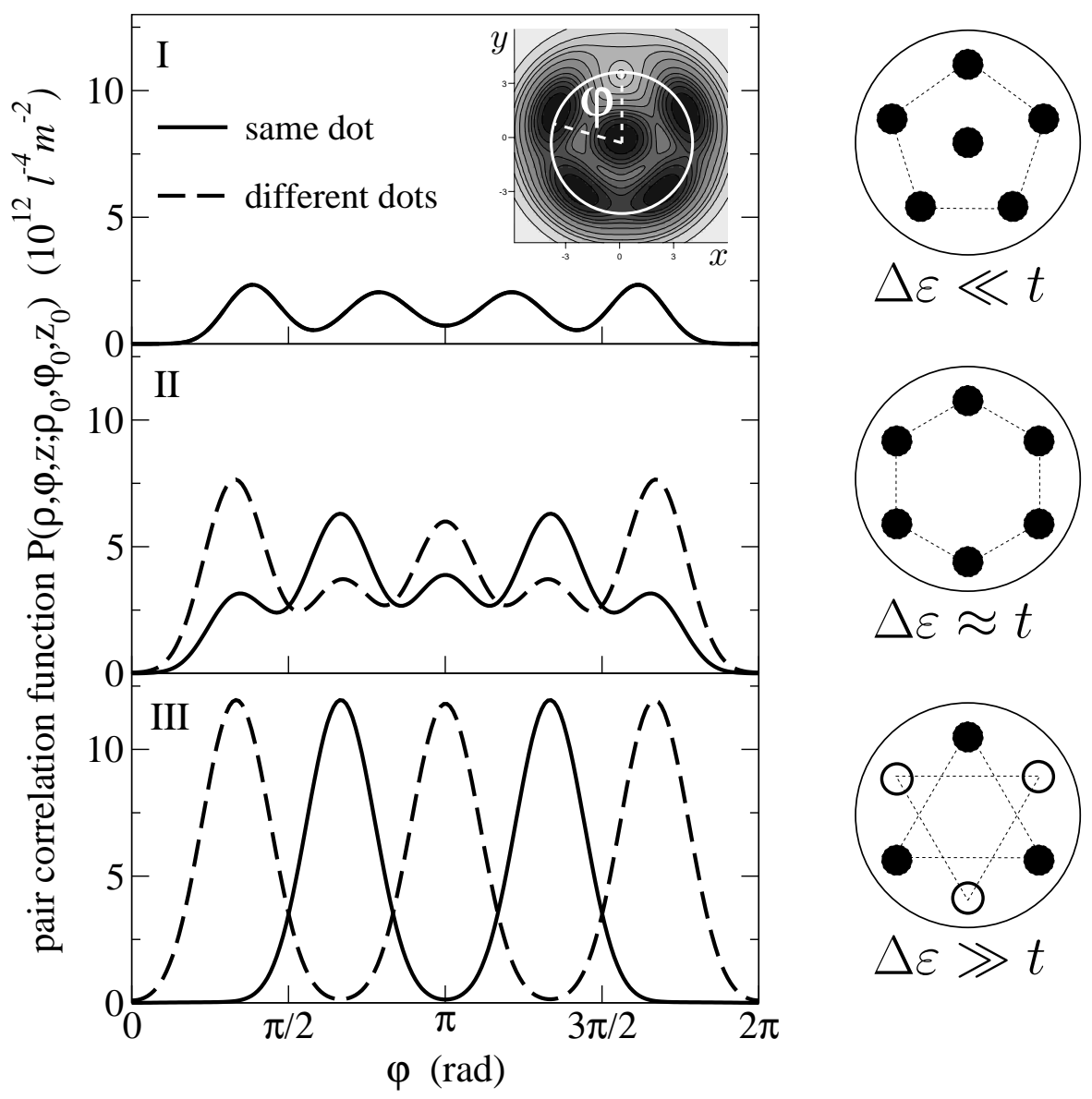

Figure 5. Angular correlation function of the three phases of Fig. 4. The three phases correspond to interdot distances $d=2,4.6,8 \mathrm{~nm}$, respectively.

To analyze the ground state of the artificial molecule in the different regimes, we show in Fig. 5 the pair correlation function $P\left(\varrho, z ; \varrho_{0}, z_{0}\right)=$ $\sum_{i \neq j}\left\langle\delta\left(\varrho-\varrho_{i}\right) \delta\left(z-z_{i}\right) \delta\left(\varrho_{0}-\varrho_{j}\right) \delta\left(z_{0}-z_{j}\right)\right\rangle / N_{e}\left(N_{e}-1\right)$ (the average is on the ground state). Figure 5 shows $P\left(\varrho, z ; \varrho_{0}, z_{0}\right)$ along a circle in the same dot (solid line) or in the opposite dot (dashed line) with respect to 
the position of a reference electron, taken at the maximum of its charge density, $\left(\varrho_{0}, z_{0}\right)$. The right column shows the electron arrangement in the QDs as inferred by the maxima of $P\left(\varrho, z ; \varrho_{0}, z_{0}\right)$.

At small $d$ (Phase I) the whole system is coherent, i.e., it behaves as a unique QD; indeed, the correlation function peaks, forming the outer shell of the centered pentagon, have the same height in both QDs. At intermediate values of the tunneling energy (Phase II) the peaks, corresponding to the vertices of a regular hexagon, have different heights. Finally, when $d$ is sufficiently large (Phase III), the structure evolves into two isolated dots coupled only via Coulomb interaction; accordingly, the peaks are again of the same height, but shifted by $60^{\circ}$.

It is important to note from Fig. 5 that Phase I and III are strongly localized phases, as demonstrated by the high peak-to-valley ratio of the correlation function, and quantum fluctuations play a minor role; therefore, electron configurations are basically determined by Coulomb interactions, and have completely classical counterparts (Partoens et al., 1997). On the contrary, in Phase II tunneling fluctuations prevent electrons from localizing and the configuration has a "liquid" character. Such phase cannot be explained in term of Coulomb interactions solely and, in fact, the hexagonal arrangement shown in Fig. 5 is classically unstable. Therefore, tunneling fluctuations may induce melting of the otherwise well localized Wigner molecule in the high field regime, and induce reentrant liquid phases, as schematically indicated in Fig. 3. A discussion of the possible experimental signatures of the different phases in inelastic light scattering experiments can be found in (Rontani et al., 2002).

\subsection{Effects of an in-plane magnetic field}

Next we discuss the effect of a finite in-plane component of the field, $B_{\|}$. To show the effect on the single-particle states, let us suppose that the total field is tilted by an angle $\theta$ with respect to the growth direction, so that $B_{\|} \neq 0$. As shown in Fig. 6, with increasing $\theta$ energy levels tend to follow the FD states backward, which are shown for comparison, since of course $B_{\perp}$ decreases with increasing angle; in addition, however, the splitting between $\mathrm{S}$ and AS levels decreases. This shows that an inplane component of the field suppresses tunneling. Note that this effect is larger for higher levels. Note also that here the S/AS labelling is used for brevity; obviously, for a general field direction with respect to the tunneling direction wavefunctions do not have a well defined S/AS symmetry. It is important to stress that deviations from the FD states are expected because the energy scale associated with the in-plane field is 

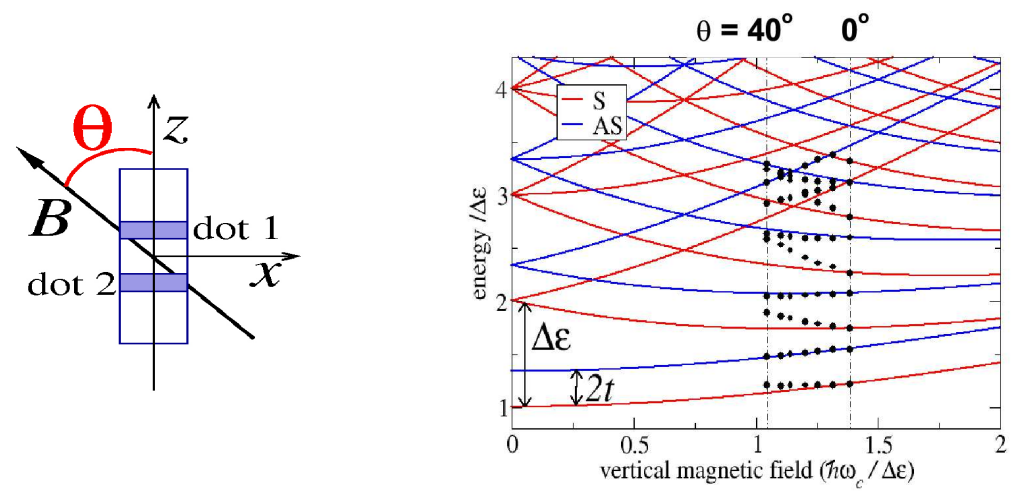

Figure 6. Evolution of single particle states in a AM when the field is rotated from the vertical direction by an angle $\theta$. Sample parameters are: $L_{w}=10 \mathrm{~nm}, d=3$ $\mathrm{nm}, V_{0}=300 \mathrm{meV}, \hbar \omega_{0}=10 \mathrm{meV}$. Solid lines represent the Fock-Darwin states $\varepsilon_{n m}^{i}$ induced by a strictly vertical field.

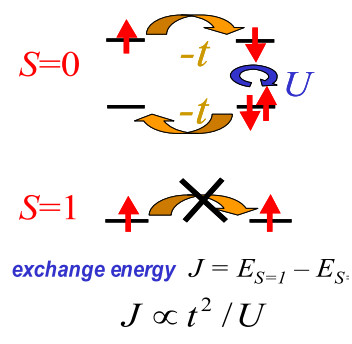

(a)

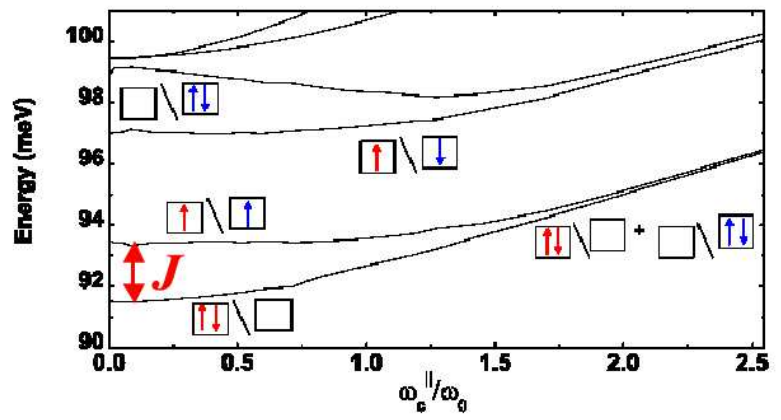

(b)

Figure \%. (a) Sketch of the energy contributions to the tunneling-induced spin-spin interaction. (b) Two-electron levels, with indication of the main component of the wavefunctions in terms of $\mathrm{S}$ (left boxes) and AS (right boxes) states. Parameters are: $L_{w}=10 \mathrm{~nm}, d=3 \mathrm{~nm}, V_{0}=300 \mathrm{meV}$, and $\hbar \omega_{0}=10 \mathrm{meV}$.

comparable to the tunneling gap; for single QDs, for example, reasonable in-plane field will not affect the FD states, since the in-plane field energy scale is typically much smaller than the single-particle gaps induced by the quantum well confinement. 
In AMs carriers sitting on either dot are not only electrostatically coupled, but also have their spin interlaced when tunneling is allowed (Burkard et al., 2000). This is sketched in Fig. 7(a). For two electrons in a singlet state it is possible to tunnel into the same dot. By doing so, they gain the tunneling energy $t$; this may compensate for the loss in the Coulomb energy $U$. This process is forbidden for two electrons in the triplet state by Pauli blocking. Different spin orderings, therefore, are associated to an exchange energy, $J$, which to lowest order in pertubation theory is $J \propto t^{2} / U$. While in real molecules $J$ is fixed by the bond length, in AMs it is possible to tune almost all energy scales by sample engineering and external fields. Since an in-plane field affects tunneling, we expect the exchange energy to be affected by an in-plane field as well.

Guided by the above considerations, we next consider the two-electron system (?; Bellucci et al., ress). At low vertical fields the ground state of single and coupled QDs is known to be a singlet state (Merkt et al., 1991; Oh et al., 1996). In the moderate field regime, therefore, the lowest energy levels are nearly unaffected by the rotation except for the shift due to the reduction of the tunneling energy, with the singlet state being the lowest.

At sufficiently high vertical field a singlet-triplet transitions take place at a given threshold field. Since the exchange energy is proportional to tunneling, we expect that the threshold fields will be lowered as $B_{\|}$ increases (Bellucci et al., ress). This is shown in Fig. 8. The singlet state is stable in the low field regime. The triplet state becomes favored in the large field regime, whether the field is perpendicular or parallel to the plane of the QD; it is should be noted, however, that this happens by different mechanisms whether $B_{\perp}$ or $B_{\|}$is large. In the former case, the squeezing of the wavefunction has a Coulomb energy cost which can only be avoided by triplet spin order. This is analogous to single QDs. However, while a finite $B_{\|}$would not affect very much electronic states in single QDs, where single-particle gaps are large, in AMs the in-plane field affects the S/AS gap. When this vanishes, no tunneling energy is lost by localizing in each dot; then the spin ordering becomes irrelevant, and the triplet state is favored only due to Zeeman energy [Fig. 7(b)].

In Figs. 7(b), 8 we also show in the insets the character of the twoelectron wavefunction of the ground state. In the low $B_{\|}$regime, the two electrons occupy only the S state, either with the $s$ symmetry with opposite spin (low field) or the $s$ and $p$ symmetry levels with the same spin orientation (high field), since a large vertical field reduces the $s-p$ gaps. In the large $B_{\|}$regime, on the contrary, $\mathrm{S}$ and $\mathrm{AS}$ states become degenerate, and are equally occupied by the two electrons, due to Coulomb correlations. 


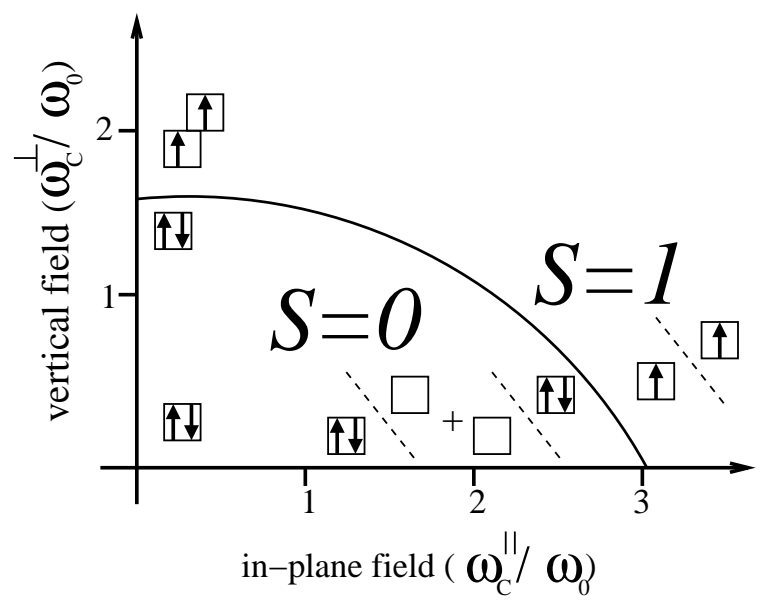

Figure 8. Singlet-triplet phase diagram calculated for a GaAs AM with $L_{W}=10$ $\mathrm{nm}, d=3 \mathrm{~nm}, \hbar \omega_{0}=4 \mathrm{meV}$. The insets show the single-particle occupation in terms of $\mathrm{S}$ (left to the dashed lines) and AS orbitals (right to the dashed lines). At low $B_{\|}$ only S orbitals are occupied.

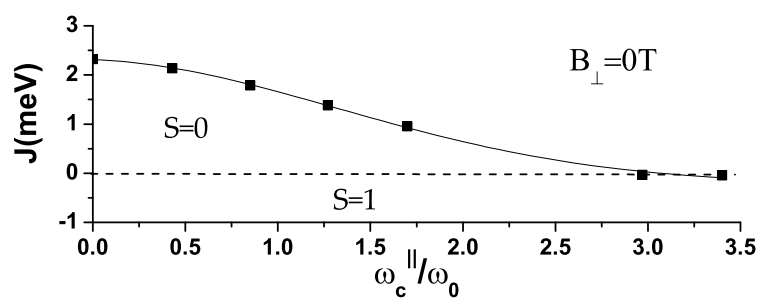

Figure 9. Exchange energy $J=E_{S=1}-E_{S=0}$ for a a GaAs AM. Same parameters as in Fig. 8.

In Fig. 9 we show the exchange energy, $J$, defined as the difference between the energy of the lowest triplet and the singlet levels, as a function of the in-plane field at zero vertical field. This is positive (i.e., the singlet is the ground state) at low fields, but rapidly decreases as the field increases. At large fields, the exchange energy changes sign, being eventually dominated by Zeeman energy.

\section{Acknowledgements}

This work was supported in part by MIUR-FIRB Quantum phases of ultra-low electron density semiconductor heterostructures, and by INFM I.T. Calcolo Parallelo (2003). Con il contributo del Ministero degli Affari Esteri, Direzione Generale per la Promozione e la Cooperazione Culturale. 


\section{References}

Ashoori, R. (1996). Nature (London), 379:413.

Bellucci, D., Rontani, M., Goldoni, G., Troiani, F., and Molinari, E. (in press). Spinspin interaction in artificial molecules with in-plane magnetic field. Physica E.

Blick, R. H., Pfannkuche, D., Haug, R. J., Klitzing, K. v., and Eberl, K. (1998). Phys. Rev. Lett., 80:4032.

Brodsky, M., Zhitenev, N. B., Ashoori, R. C., Pfeiffer, L. N., and West, K. W. (2000). Phys. Rev. Lett., 85:2356.

Bryant, G. W. (1987). Phys. Rev. Lett., 59:1140.

Burkard, G., Seelig, G., and Loss, D. (2000). Spin interactions and switching in vertically tunnel-coupled quantum dots. Phys. Rev. B, 62:2581.

Cronenwett, S. M., Oosterkamp, T. H., and Kouwenhoven, L. P. (1998). Science, 281:540.

Goldhaber-Gordon, D. G., Shtrikman, H., Mahalu, D., Abusch-Magder, D., Meirav, U., and Kastner, M. A. (1998). Nature (London), 391:156.

Jacak, L., Hawrylak, P., and Wójs, A. (1998). Quantum dots. Springer, Berlin.

Kastner, M. A. (1993). Phys. Today, 46:24.

Kouwenhoven, L. P., Hekking, F. W. J., van Wees, B. J., Harmans, C. J. P. M., Timmering, C. E., and Foxon, C. T. (1990). Phys. Rev. Lett., 65:361.

Laughlin, R. B. (1983). Phys. Rev. B, 27:3383.

Lehoucq, R. B., Maschhoff, K., Sorensen, D. C., and Yang, C. ARPACK computer code. Available at http://www. caam.rice.edu/software/ARPACK/.

Livermore, C., Crouch, C. H., Westervelt, R. M., Campman, K. L., and Gossard, A. C. (1996). Science, 274:1332.

Maksym, P. A. and Chakraborty, T. (1990). Phys. Rev. Lett., 64:108.

Maksym, P. A., Imamura, H., Mallon, G. P., and Aoki, H. (2000). J. Phys.: Condens. Matter, 12:R299.

Merkt, U., Huser, J., and Wagner, M. (1991). Energy spectra of two electrons in a harmonic quantum dot. Phys. Rev. B, 43:7320.

Oh, J. H., Chang, K. J., Ihm, G., and Lee, S. J. (1996). Electronic structure and optical properties of coupled quantum dots. Phys. Rev. B, 53:R13264.

Partoens, B., Schweigert, V. A., and Peeters, F. M. (1997). Phys. Rev. Lett., 79:3990.

Pfannkuche, D., Gudmundsson, V., and Maksym, P. A. (1993). Comparison of a hartree, a hartree-fock, and an exact treatment of quantum-dot helium. Phys. Rev. B, 47:2244-2250.

Pi, M., Emperador, A., Barranco, M., Garcias, F., Muraki, K., Tarucha, S., and Austing, D. G. (2001). Dissociation of vertical semiconductor diatomic artificial molecules. Phys. Rev. Lett., 87:066801.

Rontani, M., Goldoni, G., Manghi, F., and Molinari, E. (2002). Raman signatures of classical and quantum phases in artificial molecules. Europhys. Lett., 58:555.

Rontani, M., Goldoni, G., and Molinari, E. (2003). New directions in mesoscopic physics (towards nanoscience). NATO ASI series B: physics. Kluwer, Dordrecht. see also cond-mat/0212626.

Rontani, M., Troiani, F., Hohenester, U., and Molinari, E. (2001). Quantum phases in artificial molecules. Solid State Commun., 119:309-321. Special Issue on Spin Effects in Mesoscopic Systems. 
Szafran, B., Bednarek, S., and Adamowski, J. (2003). Correlation effects in vertical gated quantum dots. Phys. Rev. B, 67(11):115323.

Tarucha, S., Austing, D. G., Honda, T., van der Hage, R. J., and Kouwenhoven, L. P. (1996). Phys. Rev. Lett., 77:3613.

Troiani, F., Hohenester, U., and Molinari, E. (2000). Exploiting exciton-exciton interactions in semiconductor quantum dots for quantum-information processing. Phys. Rev. B, 62:RC2263.

Troiani, F., Hohenester, U., and Molinari, E. (2002). Electron-hole localization in coupled quantum dots. Phys. Rev. B, 65:RC161301. 